\section{Fatores de risco associados à mortalidade de recém-nascidos de muito baixo peso na cidade de Botucatu, São Paulo, no período 1995-2000}

\section{Risk factors for mortality in very low birth- weight infants in the city of Botucaty, State of São Paulo, during the period 1995-2000}

Maria Laura H. Prigenzi 1

Cleide E.P. Trindade 2

Ligia M. S. S. Rugolo 3

Liciana V.A. Silveira 4
Abstract

Objectives: to evaluate perinatal care, the incidence of diseases, and mortality during hospitalization and associated risk factors in very low birthweight infants (VLBW).

Methods: a cross sectional survey comparing two periods: 1995-1997 and 1998-2000, including all live-born $V L B W$ preterm infants $(n=451)$, delivered at a level III perinatal center in the city of Botucatu, State of São Paulo, Brazil. The antenatal and postnatal risk factors were analyzed using multivariable techniques.

Results: mortality decreased from $36.2 \%$ to $29.5 \%$. The survival of infants $750 \mathrm{~g}$, and 28 weeks' gestation improved and was higher than $50 \%$. The use of antenatal corticosteroid increased from $25 \%$ to $42 \%$, surfactant therapy from $14 \%$ to $28 \%$, and the incidence and severity of respiratory distress syndrome decreased. Regression analysis showed that severe respiratory distress syndrome (Odds ratio $=18)$ and early-onset sepsis (Odds ratio=2.8) were important risk factors for death in 1995-1997. During 1998-2000 early- and late-onset sepsis (Odds ratio $=10.5$ and 12 respectively) increased the risk of death.

Conclusions: the improvement in perinatal care has reduced the mortality of $V L B W$ infants. The increase in antenatal corticosteroid exposure has reduced the severity of respiratory distress syndrome. In 1998-2000, sepsis was the only risk factor associated with death.

Key words Infant, very low birth weight, Mortality, Perinatal care

\section{Resumo}

Objetivos: avaliar as práticas assistenciais, a ocorrência de doenças, a mortalidade durante a hospitalização e os fatores associados em recém-nascidos prematuros de muito baixo peso (PT-MBP).

Métodos: estudo transversal comparando dois períodos: 1995-1997 e 1998-2000 e envolvendo todos os PT-MBP nascidos vivos $(n=451)$, em um centro perinatal, em Botucatu, São Paulo, Brasil. Os fatores de risco pré-natal e pós-natal foram submetidos a análise multivariada.

Resultados: a mortalidade diminuiu de 36,2\% para 29,5\%. A sobrevida melhorou e foi superior a $50 \%$ a partir de 28 semanas e de $750 \mathrm{~g}$ de peso. O uso de corticosteróide antenatal aumentou de $25 \%$ para $42 \%$, o surfactante exógeno de $14 \%$ para $28 \%$, com redução na incidência e gravidade da síndrome do desconforto respiratório. A regressão logística mostrou que a síndrome do desconforto respiratório grave, Odds ratio $=18$, e a sepse precoce, Odds ratio=2,8, foram importantes fatores de risco para morte em 1995-1997. No periodo de 1998-2000, a sepse precoce e tardia, Odds ratio $=10,5$ e 12, respectivamente, aumentaram o risco de morte.

Conclusões: a melhora na assistência perinatal diminuiu a mortalidade do PT-MBP. O aumento na exposição antenatal ao corticosteróide diminuiu a gravidade da síndrome do desconforto respiratório. Em 19982000, a sepse foi o único fator de risco para morte.

Palavras-chave Recém-nascido de muito baixo peso, Mortalidade, Assistência perinatal 


\section{Introdução}

A melhora nos cuidados ofertadas às gestantes e aos recém-nascidos de alto risco reduziram, notadamente, a mortalidade de recém-nascidos prematuros de muito baixo peso (PT-MBP) nos serviços com unidades de terapia intensiva. Entre os avanços que mais contribuíram para a redução da mortalidade e da morbidade destacam-se a administração de surfactante exógeno no tratamento da síndrome de desconforto respiratório e o uso de corticosteróide antenatal nas gestantes em risco de parto prematuro. 1,2

Em países desenvolvidos cada vez mais sobrevivem prematuros com idades gestacionais e pesos extremamente baixos, sendo referidas taxas de sobrevida superiores a $90 \%$ para prematuros com pesos entre $1000-1500 \mathrm{~g} ; 86 \%$ para os que pesam entre $751-1000 \mathrm{~g} ; 54-70 \%$ para os menores que 750 g3,4 e até $37 \%$ de sobrevida para prematuros de $500 \mathrm{~g}$ ou menos, com nítido limite de viabilidade em 24 semanas de gestação. 5 Um estudo na Noruega mostrou sobrevida de $16-39 \%$ com 23 semanas de idade gestacional, aumentando para $44-60 \%$ com 24 semanas; $66-80 \%$ com 25 semanas; $72-84 \%$ com 26 semanas e em torno de $90 \%$ a partir de 27 semanas. 6 Entretanto, as taxas de sobrevida variam conforme o grau de desenvolvimento do país, os recursos tecnológicos disponíveis e o grau de investimento obstétrico e neonatal em gestações no limite da viabilidade.7-9

Um aspecto preocupante é que à medida que sobrevivem prematuros de extremo baixo peso e baixa idade gestacional, aumentam os riscos de doenças e complicações relacionadas à prematuridade extrema, com conseqüente prolongamento do tempo de internação, aumento no custo da assistência e ônus financeiro, emocional e social para a família. 10

A avaliação da mortalidade de recém-nascidos de muito baixo peso é importante do ponto de vista epidemiológico, pois traduz, indiretamente, a qualidade do atendimento ministrado nas Unidades Neonatais. É necessário que os serviços avaliem periodicamente suas estatísticas de mortalidade de prematuros e reportem-se aos serviços de referência nacionais ou internacionais como um padrão a ser atingido. É também importante que as unidades neonatais conheçam e avaliem criticamente a assistência ministrada e os resultados obtidos quanto à morbimortalidade dos PT-MBP, a fim de que ações sejam implantadas visando aumentar a sobrevida, reduzir as complicações da prematuridade e melhorar o prognóstico no curto e longo prazo.
Assim, este estudo teve como objetivo avaliar em recém-nascidos prematuros de muito baixo peso as práticas assistenciais, a ocorrência de doenças, a mortalidade durante a hospitalização e os fatores associados, comparando dois períodos: 1995-1997 e 1998-2000.

\section{Métodos}

Estudo do tipo transversal, no qual foram incluídos todos os recém-nascidos prematuros de muito baixo peso, ou seja com idade gestacional menor que 37 semanas e peso de nascimento inferior a $1500 \mathrm{~g}$, nascidos vivos no Hospital das Clínicas da Faculdade de Medicina de Botucatu da Universidade Estadual Paulista (UNESP), estado de São Paulo, Brasil, no período de 1995 a 2000. Não houve limite mínimo de peso, nem qualquer critério de exclusão. Os dados de interesse para o estudo foram coletados nos prontuários dos recém-nascidos após aprovação pelo Comitê de Ética da Instituição.

Foram obtidas informações sobre a assistência pré-natal e pós-natal, incluindo: doenças na gestação, uso de corticosteróide antenatal, tipo de parto, uso de ventilação mecânica e de surfactante no recém-nascido. Dos recém-nascidos obtiveram-se os dados: idade gestacional (estimada pela data da última menstruação ou ultra-sonografia antes de 20 semanas de gestação), peso de nascimento, adequação do peso para a idade gestacional conforme o percentil 10 da curva de Alexander et al., 11 escores de Apgar no primeiro e quinto minutos de vida, morbidade durante a internação, idade na alta ou óbito. O peso de nascimento e a idade gestacional foram avaliados quanto à média, desvio padrão, mínimo, máximo e também foram estratificados em faixas: de 250 gramas para o peso e de três semanas para a idade gestacional, a fim de investigar se houve mudança no perfil da população nos dois períodos de estudo, bem como para avaliar de forma mais detalhada a influência dessas variáveis na mortalidade.

A morbidade foi caracterizada pela presença das seguintes doenças: síndrome do desconforto respiratório, classificada em quatro graus de gravidade conforme critério radiológico de Morrison;12 persistência de canal arterial com repercussão hemodinâmica, diagnosticado pelo ecocardiograma; sepse clínica (podendo a hemocultura ser negativa), precoce quando manifesta nos primeiros três dias de vida e tardia após o terceiro dia; hemorragia periintraventricular, diagnosticada pelo ultra-som transfontanelar; enterocolite necrosante, caracterizada 
pelo critério clínico-radiológico de Walsh e Kliegman;13 displasia broncopulmonar, definida pela dependência de oxigênio aos 28 dias de vida, associada a sinais clínicos e radiológicos de comprometimento pulmonar. ${ }^{14}$

Os desfechos de interesse foram: alta hospitalar ou óbito.

A fim de determinar se ocorreram mudanças no período de estudo, todas as variáveis foram analisadas comparando-se dois períodos: 1995-1997 e 1998-2000.

Foi realizada análise univariada empregando-se o teste $t$ de Student para comparação entre as médias da idade gestacional e do peso de nascimento; para o estudo de associação entre as variáveis categóricas classes de idade gestacional, de peso ao nascer, freqüência de doenças e de procedimentos nos dois períodos de estudo - utilizou-se o teste do Quiquadrado; e para estimar a magnitude da associação de variáveis pré-natais, de nascimento e neonatais com a ocorrência de óbito foi efetuado o cálculo da Odds ratio com Intervalo de Confiança a 95\%.

Para avaliar a influência de diversas variáveis na ocorrência dos óbitos nos PT-MBP, as variáveis significantes na análise univariada foram submetidas à análise multivariada e ajustados modelos de regressão logística, controlados pela idade gestacional e pelos períodos: 1995-1997 e 1998-2000.

Em todas as análises o nível de significância foi de $5 \%$.

\section{Resultados}

Dentre os 8514 nascidos vivos (NV) no serviço entre primeiro de janeiro de 1995 e 31 de dezembro de 2000 , houve 451 prematuros de muito baixo peso, assim distribuídos: $210 / 4052 \mathrm{NV}$ no período de 1995-1997 e 241/4462 NV no período de 1998-2000.

No período de 1995-1997, o peso de nascimento variou entre 330 e $1490 \mathrm{~g}$, com média de $1064 \pm$ $286 \mathrm{~g}$, a idade gestacional mínima foi de 22 semanas e máxima de 36 semanas, com média de 30,2 \pm 3 semanas. No período de 1998-2000, a média do peso foi $1142 \pm 255$ g, com variação de 430 a 1495 g, e a idade gestacional média foi de 30,5 $\pm 2,5$ semanas, com variação de 23 a 36 semanas. Não houve diferença significativa entre os dois períodos.

A Tabela 1 apresenta a distribuição dos PT-MBP conforme as classes de idade gestacional e de peso ao nascer. A comparação entre os dois períodos de estudo mostrou aumento significativo da proporção de recém-nascidos na classe de 28-30 semanas de idade gestacional e diminuição na proporção dos
Tabela 1

Distribuição dos recém-nascidos prematuros de muito baixo peso (PT-MBP) conforme as classes de idade gestacional e de peso de nascimento. Botucatu, São Paulo, nos períodos de 1995-1997 e 1998-2000.

\begin{tabular}{|c|c|c|c|c|c|}
\hline \multirow[t]{2}{*}{ Classes } & \multicolumn{2}{|c|}{$\begin{array}{c}1995-1997 \\
n=210\end{array}$} & \multicolumn{2}{|c|}{$\begin{array}{c}1998-2000 \\
n=241\end{array}$} & \multirow[t]{2}{*}{$p^{*}$} \\
\hline & $\mathrm{n}$ & $\%$ & $\mathrm{n}$ & $(\%)$ & \\
\hline \multicolumn{6}{|c|}{ Idade gestacional (semanas) } \\
\hline$\leq 24$ & 15 & 7,0 & 12 & 5,0 & 0,334 \\
\hline $25-27$ & 25 & 12,0 & 31 & 13,0 & 0,758 \\
\hline $28-30$ & 55 & 26,0 & 86 & 36,0 & 0,030 \\
\hline $31-33$ & 101 & 48,0 & 97 & 40,0 & 0,094 \\
\hline $34-36$ & 11 & 5,0 & 12 & 5,0 & 0,901 \\
\hline \multicolumn{6}{|c|}{ Peso nascimento $(\mathrm{g})$} \\
\hline$<750$ & 39 & 19,0 & 28 & 12,0 & 0,038 \\
\hline $750-999$ & 43 & 20,0 & 54 & 22,0 & 0,619 \\
\hline $1000-1249$ & 56 & 27,0 & 69 & 29,0 & 0,642 \\
\hline $1250-1499$ & 72 & 34,0 & 90 & 37,0 & 0,499 \\
\hline
\end{tabular}

* p-valor do teste Qui-quadrado

Tabela 2

Mortalidade específica, mortalidade neonatal e seus componentes precoce e tardio, mortalidade pós-neonatal dos recém-nascidos prematuros de muito baixo peso (PT-MBP). Botucatu, São Paulo, nos períodos de 1995-1997 e 1998-2000.

\begin{tabular}{|c|c|c|c|c|c|}
\hline \multirow[t]{2}{*}{ Mortalidade } & \multicolumn{2}{|c|}{$\begin{array}{c}1995-1997 \\
n=210\end{array}$} & \multicolumn{2}{|c|}{$\begin{array}{c}1998-2000 \\
n=241\end{array}$} & \multirow[t]{2}{*}{$p^{*}$} \\
\hline & $\mathrm{n}$ & $\%$ & $\mathrm{n}$ & $(\%)$ & \\
\hline Específicat & 76 & 36,2 & 71 & 29,5 & 0,156 \\
\hline Neonatal (<28 dias) & 68 & 32,4 & 52 & 21,6 & 0,013 \\
\hline Neonatal precoce (<7 dias) & 55 & 26,2 & 40 & 16,6 & 0,017 \\
\hline Neonatal tardia (7-27 dias) & 13 & 6,2 & 12 & 5,0 & 0,723 \\
\hline Pós-neonatal ( $\geq 28$ dias) & 8 & 3,8 & 19 & 7,9 & 0,105 \\
\hline
\end{tabular}

* p-valor do teste Qui-quadrado; † Mortalidade específica = número de óbitos de PT-MBP em relação ao seu total

menores que $750 \mathrm{~g}$, no período de 1998-2000. Nas demais classes de peso e idade gestacional as proporções mantiveram-se estáveis nos dois períodos.

Dos 451 PT-MBP estudados, 147 (32,6\%) evoluíram para óbito. A maioria dos óbitos $(65 \%)$ ocorreu no período neonatal precoce $(<7$ dias de vida), sendo metade destes nas primeiras 24 horas de vida. Ao se comparar os dois períodos, conforme mostra a Tabela 2, a mortalidade específica dos 


\section{Figura 1}

Distribuição do percentual de óbitos dos recém-nascidos prematuros de muito baixo peso (PT-MBP), conforme as classes de idade gestacional. Botucatu, São Paulo, nos períodos de 1995-1997 e 1998-2000.

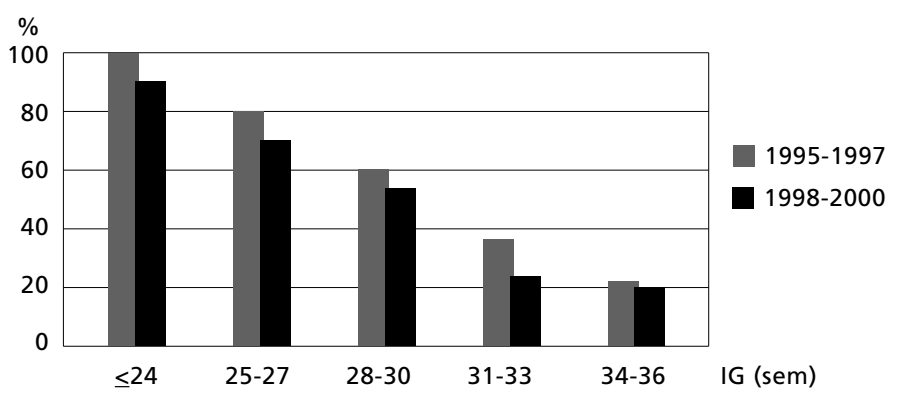

* p-valor do do teste Qui-quadrado

\section{Figura 2}

Distribuição do percentual de óbitos dos recém-nascidos prematuros de muito baixo peso (PT-MBP), conforme as classes do peso de nascimento. Botucatu, São Paulo, nos períodos de 1995-1997 e 1998-2000.

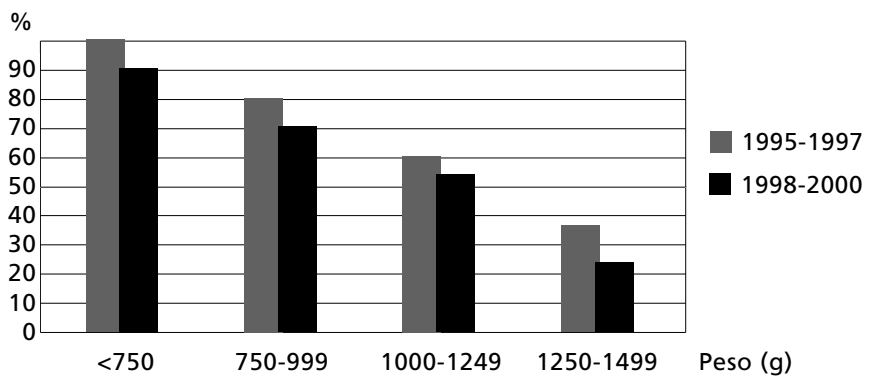

* p-valor do do teste Qui-quadrado

Figura 3

Distribuição da frequência de doenças dos recém-nascidos prematuros de muito baixo peso (PT-MBP). Botucatu, São Paulo, nos períodos de 1995-1997 e $1998-2000$

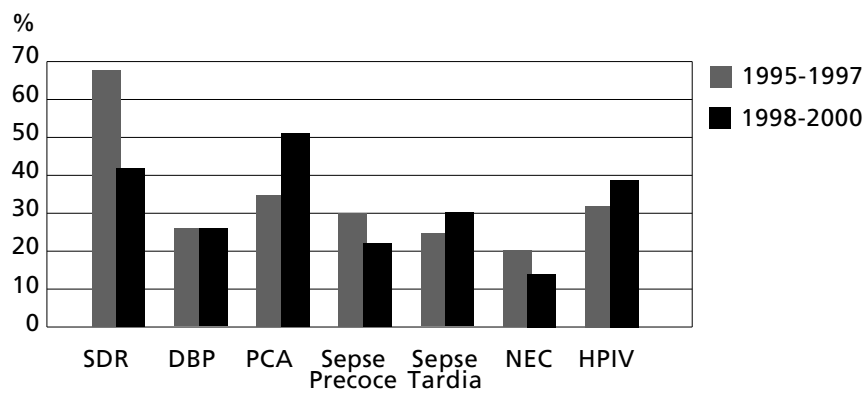

SDR = Síndrome do Desconforto Respiratório; DBP = Displasia Broncopulmonar; PCA = Persistência do canal arterial; NEC = Enterocolite necrosante; HPIV = Hemorragia peri-intraventricular. prematuros de muito baixo peso (número de óbitos de PT-MBP em relação ao seu total) diminuiu, embora sem significância estatística. Houve redução significativa na mortalidade neonatal, às custas de seu componente de mortalidade neonatal precoce. $\mathrm{O}$ percentual de óbitos no período pós-neonatal dobrou, mas esse aumento não atingiu significância estatística (Tabela 2).

A Figura 1 apresenta a distribuição do percentual de óbitos dos PT-MBP conforme as classes de idade gestacional, nos dois períodos de estudo. Em 19982000 houve redução da mortalidade em todas as classes, porém com significância estatística apenas entre 31-33 semanas $(p=0,027)$. Abaixo de 25 semanas houve apenas um sobrevivente no segundo período.

A mortalidade de acordo com as classes de peso ao nascer não decresceu no segundo período de estudo, como se observa na Figura 2. A comparação entre os dois períodos pelo teste do Qui-quadrado não mostrou redução significativa da mortalidade nas diversas classes do peso de nascimento.

A Figura 3 mostra a freqüência das principais doenças nos PT-MBP nos dois períodos de estudo. Pelo teste do Qui-quadrado verificou-se redução significativa da síndrome do desconforto respiratório $(\mathrm{p}<0,001)$ e aumento da persistência do canal arterial $(\mathrm{p}=0,003)$. A displasia broncopulmonar ocorreu em $24 \%$ nos dois períodos, e a variação nas demais doenças não foi significante.

Nas Tabelas 3 e 4 são apresentados os fatores associados à mortalidade dos PT-MBP com o cálculo da Odds ratio e Intervalo de Confiança 95\%. Na Tabela 3 observa-se que, nos dois períodos estudados, a bolsa rota por mais que 18 horas e o uso de corticosteróide antenatal não se associaram ao óbito, enquanto a variável pequeno para a idade gestacional diminuiu o risco de óbito. Destaca-se, nessa Tabela, o significativo aumento no uso de corticóide antenatal, que passou de $26 \%$ (51/194) no primeiro período, para 42\% (94/222) no segundo período $(\mathrm{p}<0,001)$.

$\mathrm{Na}$ Tabela 4 verifica-se que a mortalidade relacionada com as doenças dos recém-nascidos foi elevada, principalmente para a SDR e a sepse precoce, que aumentaram significativamente o risco de óbito dos PT-MBP nos dois períodos de estudo. Nos recém-nascidos com hemorragia peri-intraventricular o óbito foi mais freqüente no primeiro período, enquanto a sepse tardia foi fator de risco para óbito no segundo período. O surfactante exógeno foi usado em 27 de 194 PT-MBP (14\%) no primeiro período e em 62 de 222 pacientes (28\%) no segundo período, sendo esse aumento significante $(\mathrm{p}<0,001)$. 
Tabela 3

Risco de óbito dos recém-nascidos prematuros de muito baixo peso (PT-MBP) segundo variáveis de pré-natal e de nascimento. Botucatu, São Paulo, nos períodos de 1995-1997 e 1998-2000.

\begin{tabular}{|c|c|c|c|c|c|c|}
\hline \multirow[t]{3}{*}{ Variável } & \multicolumn{2}{|c|}{ 1995-1997 } & \multicolumn{4}{|c|}{$1998-2000$} \\
\hline & $\begin{array}{c}\text { Alta } \\
(n=126)\end{array}$ & $\begin{array}{l}\text { Óbito } \\
(n=68)\end{array}$ & $\begin{array}{l}\text { OR (IC95\%) } \\
\text { Valor de } \mathrm{p}\end{array}$ & $\begin{array}{c}\text { Alta } \\
(n=165)\end{array}$ & $\begin{array}{l}\text { Óbito } \\
(n=57)\end{array}$ & $\begin{array}{l}\text { OR (IC95\%) } \\
\text { Valor de } p\end{array}$ \\
\hline & $\mathrm{n}$ & $\mathrm{n}$ & & $\mathrm{n}$ & $\mathrm{n}$ & \\
\hline HIG & 60 & 25 & $\begin{array}{c}0,64(0,36-1,17) \\
p=0,146\end{array}$ & 67 & 8 & $\begin{array}{c}0,24(0,11-0,54) \\
p<0,001\end{array}$ \\
\hline Bolsa rota $>18 \mathrm{~h}$ & 27 & 16 & $\begin{array}{c}0,88(0,44-1,77) \\
p=0,597\end{array}$ & 38 & 14 & $\begin{array}{c}1,10(0,54-2,23) \\
p=0,800\end{array}$ \\
\hline Corticóide antenatal & 36 & 15 & $\begin{array}{c}0,71(0,35-1,41) \\
p=0,325\end{array}$ & 73 & 21 & $\begin{array}{c}0,73(0,39-1,35) \\
p=0,318\end{array}$ \\
\hline Cesárea & 93 & 32 & $\begin{array}{c}0,34(0,18-0,63) \\
p=0,001\end{array}$ & 89 & 26 & $\begin{array}{c}0,64(0,36-1,14) \\
p=0,129\end{array}$ \\
\hline Sexo masculino & 49 & 40 & $\begin{array}{c}2,55(1,38-4,71) \\
p=0,002\end{array}$ & 83 & 43 & $\begin{array}{c}1,61(0,92-2,83) \\
p=0,096\end{array}$ \\
\hline PIG & 100 & 35 & $\begin{array}{c}0,29(0,16-0,53) \\
p<0,001\end{array}$ & 109 & 19 & $\begin{array}{c}0,20(0,11-0,37) \\
p<0,001\end{array}$ \\
\hline IG $<28$ semanas & 5 & 35 & $\begin{array}{c}22,00(8,10-59,90) \\
p<0,001\end{array}$ & 10 & 33 & $\begin{array}{c}13,9(6,30-30,60) \\
p<0,001\end{array}$ \\
\hline $\mathrm{PN}<1000 \mathrm{~g}$ & 29 & 53 & $\begin{array}{c}8,34(4,40-15,80) \\
p<0,001\end{array}$ & 31 & 51 & $\begin{array}{c}11,40(5,99-21,80) \\
p<0,001\end{array}$ \\
\hline Apgar $10 \min \leq 3$ & 32 & 49 & $\begin{array}{c}8,74(4,42-17,30) \\
p<0,001\end{array}$ & 60 & 40 & $\begin{array}{c}3,30(1,80-6,07) \\
p<0,001\end{array}$ \\
\hline Apgar $5 \circ \min \leq 6$ & 21 & 34 & $\begin{array}{c}4,91(2,49-9,68) \\
p<0,001\end{array}$ & 32 & 26 & $\begin{array}{c}5,72(2,99-10,90) \\
p<0,001\end{array}$ \\
\hline
\end{tabular}

HIG=hipertensão induzida na gestação; PIG=pequeno para idade gestacional; IG=idade gestacional; PN=peso de nascimento

Tabela 4

Risco de óbito dos recém-nascidos prematuros de muito baixo peso (PT-MBP) segundo variáveis neonatais. Botucatu, São Paulo, nos períodos de 1995-1997 e 1998-2000.

\begin{tabular}{|c|c|c|c|c|c|c|}
\hline \multirow[t]{3}{*}{ Variável } & \multicolumn{2}{|c|}{$1995-1997(n=194)$} & \multicolumn{4}{|c|}{$1998-2000(n=222)$} \\
\hline & $\begin{array}{c}\text { Alta } \\
(n=126)\end{array}$ & $\begin{array}{l}\text { Óbito } \\
(n=68)\end{array}$ & $\begin{array}{l}\text { OR (IC95\%) } \\
\text { Valor de } p\end{array}$ & $\begin{array}{c}\text { Alta } \\
(n=165)\end{array}$ & $\begin{array}{l}\text { Óbito } \\
(n=57)\end{array}$ & $\begin{array}{l}\text { OR (IC95\%) } \\
\text { Valor de } p\end{array}$ \\
\hline & $\mathrm{n}$ & $\mathrm{n}$ & & $\mathrm{n}$ & $\mathrm{n}$ & \\
\hline SDR & 71 & 57 & $\begin{array}{c}4,01(1,92-8,37) \\
p<0,001\end{array}$ & 55 & 39 & $\begin{array}{c}4,45(2,34-8,48) \\
p<0,001\end{array}$ \\
\hline PCA & 55 & 14 & $\begin{array}{c}0,33(0,17-0,66) \\
p=0,001\end{array}$ & 83 & 31 & $\begin{array}{c}1,18(0,64-2,15) \\
p=0,595\end{array}$ \\
\hline Sepse precoce & 30 & 28 & $\begin{array}{c}2,69(1,42-5,12) \\
p=0,002\end{array}$ & 22 & 26 & $\begin{array}{c}5,91(2,95-11,8) \\
p<0,001\end{array}$ \\
\hline Sepse tardia & 50 & 19 & $\begin{array}{c}1,11(0,56-2,22) \\
p=0,766\end{array}$ & 61 & 27 & $\begin{array}{c}4,60(2,09-10,20) \\
p<0,001\end{array}$ \\
\hline HPIV & 29 & 32 & $\begin{array}{c}2,97(1,58-5,59) \\
p<0,001\end{array}$ & 57 & 24 & $\begin{array}{c}1,38(0,74-2,55) \\
p=0,307\end{array}$ \\
\hline NEC & 24 & 14 & $\begin{array}{c}1,14(0,55-2,40) \\
p=0,721\end{array}$ & 20 & 13 & $\begin{array}{c}2,14(0,99-4,65) \\
p=0,051\end{array}$ \\
\hline Surfactante & 12 & 15 & $\begin{array}{c}2,69(1,18-6,14) \\
p=0,016\end{array}$ & 35 & 27 & $\begin{array}{c}3,42(1,80-6,58) \\
p<0,001\end{array}$ \\
\hline Ventilação mecânica & 60 & 61 & $\begin{array}{c}9,00(3,81-21,30) \\
p<0,001\end{array}$ & 89 & 54 & $\begin{array}{c}12,1(4,21-35,00) \\
p<0,001\end{array}$ \\
\hline
\end{tabular}

SDR=síndrome do desconforto respiratório; PCA=persistência do canal arterial; HPIV=hemorragia peri-intravetricular; $\mathrm{NEC}=$ enterocolite necrosante 
Distribuição da mortalidade dos recém-nascidos prematuros de muito baixo peso (PT-MBP) segundo a presença e o grau da síndrome do desconforto respiratório. Botucatu, São Paulo, nos períodos de 1995-1997 e 1998-2000.

\begin{tabular}{|c|c|c|c|c|c|c|c|c|}
\hline \multirow[t]{3}{*}{ SDR - Graus } & \multicolumn{4}{|c|}{$1995-1997$ * } & \multicolumn{4}{|c|}{$1998-2000 t$} \\
\hline & \multicolumn{2}{|c|}{ Alta } & \multicolumn{2}{|c|}{ Óbito } & \multicolumn{2}{|c|}{ Alta } & \multicolumn{2}{|c|}{ Óbito } \\
\hline & $\mathrm{n}$ & $(\%)$ & $n$ & $(\%)$ & $n$ & $(\%)$ & $\mathrm{n}$ & $(\%)$ \\
\hline I & 18 & 90,0 & 2 & 10,0 & 11 & 69,0 & 5 & 31,0 \\
\hline II & 38 & 69,0 & 17 & 31,0 & 20 & 64,5 & 11 & 35,5 \\
\hline III & 13 & 41,0 & 19 & 59,0 & 19 & 65,5 & 10 & 34,5 \\
\hline IV & 2 & 9,5 & 19 & 90,5 & 5 & 28,0 & 13 & 72,0 \\
\hline SDR ausente & 55 & 83,0 & 11 & 17,0 & 113 & 75,0 & 18 & 25,0 \\
\hline
\end{tabular}

$\mathrm{SDR}=$ síndrome do desconforto respiratório; * qui-quadrado=18,51; $<<0,001 ; \dagger$ qui-quadrado=22,28; $\mathrm{p}<0,001$

Tabela 6

Análise multivariada dos fatores de risco para óbito nos recém-nascidos prematuros de muito baixo peso (PT-MBP). Botucatu, São Paulo, nos períodos de 1995-1997 e 1998-2000.

\begin{tabular}{lccc}
\hline Fatores & OR & IC95\% & Valor de p \\
\hline Idade gestacional $<28$ semanas & 5,46 & $2,62-11,36$ & $<0,001$ \\
Apgar de 50 minuto $\geq 6$ & 2,04 & $1,15-3,62$ & 0,015 \\
Apgar de 50 minuto $\leq 3$ & 12,35 & $4,59-33,33$ & $<0,001$ \\
SDR grave & 2,70 & $1,90-3,85$ & $<0,001$ \\
Sepse precoce & 3,15 & $1,77-5,62$ & $<0,001$ \\
\hline
\end{tabular}

SDR=síndrome do desconforto respiratório.

Tabela 7

Análise multivariada dos fatores de risco para óbito nos recém-nascidos prematuros de muito baixo peso (PT-MBP). Botucatu, São Paulo, no período de 1995-1997 e 1998-2000.

\begin{tabular}{lccc}
\hline Fatores & OR & IC95\% & Valor de $\mathbf{p}$ \\
\hline Período 1995-1997 & & & $<0,001$ \\
$\quad$ SDR grave & 17,86 & $6,62-47,62$ & 0,001 \\
Sepse precoce & 2,76 & $1,28-5,99$ & 0,001 \\
PCA & 0,25 & $0,11-0,57$ & $<0,001$ \\
Período 1998- 2000 & & & $<0,001$ \\
$\quad$ Sepse precoce & 10,53 & $3,68-30,30$ & $3,65-38,46$ \\
$\quad$ Sepse tardia & 11,95 & & \\
\hline
\end{tabular}

SDR=síndrome do desconforto respiratório; PCA=persistência do canal arterial.

Nos dois períodos estudados, houve associação entre a presença e gravidade da síndrome do desconforto respiratório (SDR) e a mortalidade dos PTMBP, como mostra a Tabela 5.
Foram ajustados modelos de regressão logística para avaliar a influência das variáveis significativas na análise univariada, na ocorrência de óbitos dos PT-MBP. Entre os vários fatores incluídos no 
modelo, as variáveis: idade gestacional menor que 28 semanas, Apgar baixo no $5^{\circ}$ minuto, SDR grave (graus III e IV) e sepse precoce permaneceram como fatores associados ao aumento do risco de óbito, no período de 1995 a 2000 (Tabela 6).

Em seguida, as variáveis significativas na análise univariada foram controladas por períodos: 19951997 e 1998-2000 (Tabela 7). No período de 19951997, a SDR grave e a sepse precoce associaram-se ao aumento do risco de óbito, enquanto a persistência do canal arterial associou-se com diminuição do risco de morte. De 1998 a 2000, a sepse foi o único fator de risco para óbito nos PTMBP. Tanto a sepse precoce como a tardia tiveram forte influência, aumentando em 10,5 e 12 vezes, respectivamente, o risco de morte (Tabela 7).

\section{Discussão}

A diminuição na mortalidade dos PT-MBP de 36,2\% para $29,5 \%$ entre 1995 até 2000 é um dado auspicioso, principalmente ao considerar-se a redução progressiva na mortalidade desses recém-nascidos durante a última década. Em análise anterior efetuada no serviço, com a mesma metodologia, observou-se que a mortalidade passou de $42,8 \%$ no período de 1990-1993 para 35,9\% no período de 1994-1997. Essa evolução traduz os esforços na melhora dos cuidados perinatais ministrados, resultando no aumento de sobrevida, principalmente nas classes de idades gestacionais inferiores a 30 semanas, que requerem cuidados mais complexos. 15

As cifras de mortalidade no presente estudo são compatíveis às referidas em várias publicações internacionais, que mostram taxas de mortalidade de recém-nascidos de muito baixo peso variando de $10 \%$ a $30 \% .{ }^{16,17}$ No Brasil, Gomes et al. 18 documentaram $32 \%$ de mortalidade em prematuros de muito baixo peso, em hospitais públicos do Rio de Janeiro, no período de 1994-2000; e o estudo da Rede Brasileira de Pesquisas Neonatais, envolvendo oito centros, apontou taxas de mortalidade de $21 \%$ a $34 \%$ para PT-MBP, no período de 1998 a 1999.19

Redes Perinatais internacionais, reunindo vários centros e um número considerável de PT-MBP, têm mostrado estatísticas animadoras de sobrevida. $\mathrm{Na}$ série da Vermont-Oxford Network, período de 1991 a 1999, que reuniu 362 Unidades de cuidados intensivos neonatais e 118.448 recém-nascidos com pesos de 501 a $1500 \mathrm{~g}$, a mortalidade variou de $18,1 \%$ em 1991 a $14,8 \%$ em 1999. Nessa série houve importante decréscimo na morbimortalidade na primeira metade da década, entretanto, a partir de 1995 nenhum benefício adicional foi observado. 20 Ao comparar os resultados do presente estudo com essa série da Vermont-Oxford 20 e também com outros estudos recentes de países desenvolvidos $4,7,8$ fica evidente a necessidade de intensificar os investimentos na assistência aos prematuros de muito baixo peso, a fim de reduzir a mortalidade desses, que ainda é elevada em nosso meio.

Neste estudo a mortalidade neonatal no primeiro período foi de $32,4 \%$ e no segundo de $21,6 \%$, mostrando que a maioria dos óbitos ocorreu nos primeiros 27 dias de vida. Esses dados estão próximos aos maiores valores encontrados por Lemons et al., 4 cuja média foi de $14,0 \%$, variando de $7,0 \%$ a $20,0 \%$; e são discretamente inferiores à média documentada no estudo de Duarte e Mendonça,21 que avaliaram, no período de 2001-2002, em quatro maternidades do Rio de Janeiro, 487 recém-nascidos de muito baixo peso, sendo excluídos os malformados e os menores que $500 \mathrm{~g}$, e encontraram $26 \%$ de mortalidade neonatal, variando de 10 a $37 \%$ entre os hospitais.

A variação na incidência de doenças, bem como na mortalidade a elas relacionada nos dois períodos focalizados nesse estudo, refletem mudanças na assistência perinatal. Assim, a menor incidência de síndrome do desconforto respiratório em 1998-2000 pode ser conseqüente ao aumento do uso de corticosteróide antenatal, que também reduziu a gravidade dessa doença nesse período. Esses resultados são coerentes com o benefício do uso de corticosteróide antenatal, que já está consagrado em estudos de metanálise.22 O emprego de corticosteróide antenatal vem aumentando gradualmente neste serviço, de $12,6 \%$ no início da década de noventa, 15 para 49,2\% em 2000. Entretanto, esses valores ainda estão aquém dos obtidos em países desenvolvidos, ${ }^{20}$ onde as cifras atingem até $71,6 \%$, e também são inferiores aos resultados do estudo da Rede Brasileira de Pesquisas Neonatais que, em 2001 , detectou $61,3 \%$ de corticoterapia antenatal em gestações abaixo de 35 semanas. 23

O surfactante exógeno foi pouco usado em $1995-$ 1997, quando a freqüência e gravidade da síndrome do desconforto respiratório eram maiores, mas em 1998-2000 a freqüência de uso do surfactante dobrou, atingindo $28 \%$ e, embora essa porcentagem não pareça alta, deve-se considerar que nesse período $42 \%$ dos recém-nascidos tiveram tal síndrome, portanto, o surfactante foi indicado em aproximadamente dois terços dos casos. Em países desenvolvidos 20 o uso de surfactante é mais freqüente, com cifras superiores a $60 \%$.

A regressão logística mostrou que no período de 
1995 a 2000 a prematuridade extrema (idade gestacional menor que 28 semanas), a depressão neonatal grave, a síndrome do desconforto respiratório grave (graus III e IV) e a sepse precoce foram os fatores de risco para óbito nos prematuros de muito baixo peso, fatores esses que têm sido evidenciados em outros estudos da literatura. ${ }^{24-26}$ Resultados diferentes foram documentados no estudo de Duarte e Mendonça, ${ }^{21}$ que mostrou o sexo masculino, a hemorragia materna, o uso de ventilação mecânica, o peso ao nascer e o escore de risco CRIB como fatores de risco para óbito neonatal em recémnascidos de muito baixo peso. Entretanto, deve ser considerado que os fatores associados à mortalidade de recém-nascidos variam conforme as características da população estudada, as práticas assistenciais, a metodologia e o período de estudo.

Um dado interessante no presente estudo foi a variação das doenças associadas ao óbito nos dois períodos avaliados. Em 1998-2000 a síndrome do desconforto respiratório não mais se associou ao óbito, provavelmente devido ao incremento no uso de corticosteróide antenatal e de surfactante no recém-nascido, e nesse período a sepse foi o fator de risco para óbito nos prematuros de muito baixo peso, o que corrobora a importância dos quadros infecciosos na mortalidade de prematuros em UTI neonatal. 18,27,28

A associação da persistência do canal arterial com diminuição do risco de óbito deve ser vista com cautela, pois pelo fato do ecocardiograma ser realizado fora da UTI, o recém-nascido deveria estar estável para que o exame fosse realizado, assim os pacientes mais graves que faleceram na primeira semana de vida não tiveram o diagnóstico ecocardiográfico da persistência do canal arterial.

Esse estudo aponta progressos e melhorias na assistência aos prematuros de muito baixo peso, especialmente na assistência em sala de parto, na assistência respiratória, no aumento do emprego de surfactante e de corticosteróide antenatal, bem como no controle hemodinâmico dos pacientes, permitindo controlar o efeito da depressão neonatal e da síndrome do desconforto respiratório na mortalidade. O grande desafio consiste na prevenção, diagnóstico e tratamento da sepse neonatal, objetivando reduzir a mortalidade por essa causa. Chama a atenção que em 1998-2000 a sepse tardia foi fator importante na mortalidade dos prematuros de muito baixo peso, o que provavelmente se deve à maior permanência hospitalar desses prematuros e ao grande número de procedimentos invasivos a que são submetidos. 27,28

Em 2002, a Organização Mundial de Saúde estimou que $30 \%$ a $40 \%$ das mortes neonatais estavam associadas à infecção, concluindo que a infecção seria responsável por 1,4 a 1,9 milhões de mortes/ano ou 3900 a 5200 mortes/dia no mundo. ${ }^{29}$ Os resultados do presente estudo confirmam que o panorama das infecções continua sombrio para os prematuros de muito baixo peso.

Há que se considerar que quanto menor for o prematuro, mais complexa será a assistência necessária para sua sobrevivência e o tratamento em UTI neonatal tem custo elevado e acesso relativamente restrito devido à escassez de leitos, em especial nos hospitais públicos. Isso tem gerado preocupação com a relação custo-efetividade da UTI neonatal, principalmente no limite da viabilidade. 30 Assim, a avaliação crítica dos resultados obtidos em cada serviço, visando conhecer seus limites e otimizar a utilização dos recursos tecnológicos disponíveis, é de fundamental importância nos dias atuais, para garantir qualidade e disponibilidade da assistência neonatal.

Frente aos resultados deste estudo conclui-se que houve melhora na assistência aos prematuros de muito baixo peso e diminuição da mortalidade desses, nos anos 1998-2000. O aumento no uso de corticosteróide antenatal influenciou na diminuição da gravidade da síndrome de desconforto respiratório e dos óbitos a ela associados. A sepse neonatal foi o único fator de risco para óbito no período de 1998-2000. A prevenção de infecções neonatais, a ampliação no uso de corticosteróide antenatal e de surfactante exógeno em prematuros com síndrome do desconforto respiratório devem ser o foco dos investimentos para reduzir a mortalidade e conseqüentemente melhorar a expectativa quanto ao prognóstico dos prematuros de muito baixo peso.

\section{Referências}

1. Hack M, Fanaroff AA. Outcomes of children of extremely low birth weight and gestational age in the 1990s. Semin Neonatol. 2000; 5: 89-106.
2. Stoelhorst GM, Rijken M, Martens SE, Brand R, den Ouden $\mathrm{AL}$, Wit JM, Veen S. Changes in neonatology: comparison of two cohorts of very preterm infants (gestational age $<32$ weeks): the Project On Preterm and Small for Gestational Age Infants 1983 and the Leiden follow-up Project on Prematurity 1996-1997. Pediatrics. 2005; 115: 396-405. 
3. Cole FS. Extremely preterm birth defining the limits of hope. N Engl J Méd. 2000; 343: 429-30.

4. Lemons JA, Bauer CR, Oh W, Korones SB, Papile LA, Stoll BJ, Verter J, Temprosa M, Wright LL, Ehrenkranz RA, Fanaroff AA, Stark A, Carlo W, Tyson JE, Donovan EF, Shankaran S, Stevenson DK. Very low birth weight outcomes of the National Institute of Child Health and human development neonatal research network, January 1995 trough December 1996. Pediatrics. 2001; 107: 1-8.

5. Rieger-Fackeldey E, Schulze A, Pohlandt F, Schwarze R, Dinger J, Lindner W. Short-term outcome in infants with a birth weight less than 501 grams. Acta Paediatr. 2005; 94: 211-6.

6. Markestad T, Kaarsen PI, Ronnestad A, Reigstad H, Lossius K, Medbo S, Zanussi G, Engelund IE, Polit C, Skjaeerven $\mathrm{R}$, Irgens LM. Early death, morbidity, and need of treatment among extremely premature infants. Pediatrics. 2005; 115:1289-98.

7. Anthony S, den Ouden L, Brand R, Verloove-Vanhorick P, Gravenhorst JB Changes in perinatal care and survival in very preterm and extremely preterm infants in The Netherlands between 1983 and 1995. Obstet Gynecol. 2004; 112: 170-7.

8. Louis JM, Ehrenberg HM, Collin MF, Mercer BM. Perinatal intervention and neonatal outcomes near the limit of viability. Am J Obstet Gynecol. 2004; 191: 1398-402.

9. Acolet D, Elbourne D, McIntosh N, Weindling M, Korkodilos M, Haviland J, Modder J, Macintosh M. Project 27/28: inquiry into quality of neonatal care and its effect on the survival of infants who were born at 27 and 28 weeks in England, Wales, and Northen Ireland. Pediatrics. 2005; 116 : 1457-65.

10. Wilson-Costello D, Friedman H, Minich N, Fanaroff AA, Hack M. Improved survival rates with increased neurodevelopmental disability for extremely low birth weight infants in the 1990s. Pediatrics. 2005; 115: 997-1003.

11. Alexander GR, Hines JH, Kaufman RB, Mor J, Kogan M. A United States national reference for fetal growth. Obstet Gynecol. 1996; 87: 163-8.

12. Morrison SC Radiologic findings of newborn respiratory diseases. In: Carlo WA, Chatburn RL. Neonatal respiratory care. 2. ed. Chicago: Year Book Medical Publishers; 1988. p. 289-319.

13. Walsh MC, Kliegman RM Necrotizing enterocolitis: treatment based on staging criteria. Pediatr Clin North Am. 1986; 33: 179-201.

14. Bancalari EH, Abdeenour GE, Feller R, Gannon J. Bronchopulmonary dysplasia: clinical presentation. J Pediatr. 1979; 95: 819-23.

15. Corrales-Mayorga BC. Morbimortalidade de recémnascidos de muito baixo-peso (peso inferior a 1500 gramas) do Berçário do Hospital das Clínicas da Faculdade de Medicina Botucatu-UNESP, no período de 1990 a 1997 (dissertação mestrado). Botucatu: Faculdade de Medicina da Universidade Estadual Paulista; 1998.

16. Velaphi SC, Mokhachane M, Mphahlele RM, BeckhArnold E, Kuwanda ML, Cooper PA. Survival of very-lowbirth-weight infants according to birth weight and gestational age in a public hospital. S Afr Med J. 2005; 95: 5049 .

Recebido em 2 de setembro de 2006

Versão final apresentada em 10 de junho de 2007

Aprovado em 26 de setembro de 2007
17. Hoyert DL, Freedman MA, Strobino DM, Guyer B. Annual summary of vital statistics - 2000. Pediatrics. 2001; 108: 1241-55.

18. Gomes MASM, Lopes JMA, Moreira MEL, Gianini NOM. Assistência e mortalidade neonatal no setor público do Município do Rio de Janeiro, Brasil: uma análise do período 1994/2000. Cad Saúde Pública. 2005; 21: 1269-77.

19. Leone CR, Sadeck LSR, Vaz FC, Almeida MFB, Draque MC, Guinsburg R, Marba S, Neto AA, Martinez FE, Pinhata MM, Lopes JM, Bonfim O, Procianoy RS, Bejamin $\mathrm{AC}$, Petean CE, Prigenzi MLH, Fiori RM, Fiori JHL. Brazilian neonatal research network (BNRN): very-lowbirth-weight (VLBW) infant morbidity and mortality. Pediatr Res. 2001; 49: 405A.

20. Horbar JD, Badger GJ, Carpenter JH, Fanaroff AA, Kilpatrick S, Lacorte M, Phibbs R, Soll RF. Trends in mortality and morbidity for very low birth weight infants, 1991-1999. Pediatrics. 2002; 110: 143-51.

21. Duarte JLB, Mendonça GAS. Fatores associados à morte neonatal em recém-nascidos de muito baixo peso em quatro maternidades no Município do Rio de Janeiro. Cad Saúde Pública. 2005; 21: 181-91.

22. Crowley P. Prophylatic corticosteroids for preterm birth (Cochrane review). Cochrane Database Syst Rev. 2002; (3): CD000065.

23. Rede Brasileira de Pesquisas Neonatais. Uso antenatal de corticosteróide e evolução clínica de recém-nascidos prétermo. J Pediatr (Rio J). 2004; 80: 277-84.

24. Araújo BF, Bozzetti MC, Tanaka ACA. Mortalidade neonatal precoce no Município de Caxias do Sul: um estudo de coorte. J Pediatr (Rio J). 2000; 76: 200-6

25. Garcia Arias MB, Zuluaga Arias P, Arrabal Teran MC Arizcun Pineda J. Risk factors for mortality in very low birth weight infants with respiratory distress syndrome. An Pediatr (Barc). 2005; 63: 109-15.

26. Weirich CF, Andrade ALSS, Turchi MD, Silva SA, MoraisNeto OL, Minamisava R, Marques SM. Neonatal mortality in intensive care units of Central Brazil. Rev Saúde Publica. 2005; 39: 775-81.

27. Rugolo LMSS, Bentlin MR, Curi PR, Rugolo Junior A, Hashimoto M. A function to predict the risk for death in the neonatal sepsis. In: Voto LS, Margulies M, Cosme EV, editors. IV World Congress of Perinatal Medicine; 1999 Abr. 7-22; Buenos Aires, Argentina. Bologna: Monduzzi; 1999. p. 647-51.

28. Stool BJ, Hansen N, Fanaroff AA, Wright LL, Carlo W, Ehrenkranz RA, Lemons JA, Donovan EF, Stark A, Tyson JE, Oh W, Bauer CR, Korones SB, Shankaran S, Laptook AR, Stevenson DK, Pappile LA, Poole WK. Late-onset sepsis in very low birth weight neonates: the experience of the NICHD Neonatal Network. Pediatrics. 2002; 110: 28591.

29. WHO (World Health Organization). Maternal and child health. Available from : http:/www.who.int/das/cat98/ mat8.htm\#. [2002 July 20].

30. Stolz JW, McCormick MC. Restricting access to neonatal intensive care: effect on mortality and economic savings. Pediatrics. 1998; 101: 344-8. 